\title{
Docetaxel plus ADT significantly improves patient outcomes
}

Androgen-deprivation therapy (ADT) has, for more than 70 years, been the mainstay of treatment for patients with metastatic prostate cancer. Now, results of a phase III clinical trial reveal that addition of docetaxel to routinely used ADT regimens results in a significant improvement in overall survival compared with use of ADT alone.

\section{Patients in the docetaxel plus ADT arm had a significant increase in overall survival... 77}

Explaining the reasons for adding docetaxel to ADT regimens, lead author Christopher Sweeney explains "resistance to ADT often occurs after about 18 months and this is called castration-resistant prostate cancer (CRPC). Docetaxel was the first agent to prolong overall survival when used to treat CRPC."

To test the efficacy of ADT plus docetaxel versus ADT, investigators enrolled a total of 790 patients with metastatic prostate cancer. Patients were assigned to receive either ADT with 6 cycles of docetaxel $\left(75 \mathrm{mg}\right.$ per $\mathrm{m}^{2}$ of body-surface area every 3 weeks) or ADT alone. Patients in the docetaxel plus ADT arm had a significant increase in overall survival compared with those who received ADT alone (median overall survival 57.6 months versus 44.0 months). Patients who received ADT plus docetaxel also had a significantly longer median time to progression, as defined by biochemical, symptomatic or radiographic criteria. Patients in the ADT plus docetaxel group were also significantly more likely to have a decline in serum PSA to undetectable levels after 12 months. Furthermore, grade 3 or 4 febrile neutropenia, which was the most frequently observed adverse event, occurred in only $6.2 \%$ of patients in the ADT plus docetaxel group.

Taken together, these findings suggest that the addition of docetaxel to ADT regimens represents a meaningful improvement in the treatment of patients with metastatic prostate cancer: overall survival is extended, with a significant increase in progression-free survival, with few adverse events. A formal quality-of-life analysis is currently awaited.

Concluding the findings of this study, Sweeney highlights: "This is the first study to show an improvement in overall survival in this setting and the first time any drug tested in patients with prostate cancer increased the median survival by more than 12 months." Reflecting on how these findings might improve clinical practice, Sweeney adds, "patients with metastatic, hormone-sensitive prostate cancer who are fit for chemotherapy should be offered docetaxel after being counselled on the risks and benefits of this approach."

\section{Peter Sidaway}

Original article Sweeney, C. J. et al. Chemohormonal therapy in metastatic hormone-sensitive prostate cancer. N. Engl. J. Med. doi:10.1056/NEJMoa1503747 